\title{
A Preliminary Study: The Novel AI Voice-Recognition System for Acute Care Hospitals in the United States
}

\author{
Meng Yin Wu, Wen Ta Chiu*, Yu-Chuan Jack Li, Wen-Shan Chien, Jonathan Wu, Rachele Hwong, $\square$ \\ Stewart, PingYi Lin, Claire Huang, Su Yen Wu and Corey Teng \\ AHMC Healthcare, USA \\ Taipei Medical University, Taiwan \\ *Corresponding author: Wen-Ta, Chiu AHMC Healthcare USA 500 East Main St Alhambra CA 91801
}

\begin{abstract}
ARTICLE INFO
Received: 㣰 February 26, 2020

Published: 慧 March 04, 2020

Citation: Meng Y W, Wen T C, Jonathan W, Rachele H, JJ Stewart etal., Gamification in Late-Life Depression: A Serious Game for a Serious Problem. Biomed J Sci \& Tech Res 26(2)-2020. BJSTR. MS.ID.004321.
\end{abstract}

Keywords: Artificial Intelligence; Voice Smart Care; Patient Satisfaction; Patient safety; Quality of Care; Machine Learning; Healthcare IT

\section{ABSTRACT}

Background: The inspiration for the VSC system came from clinical and personal insights over the past 20-30 years. The VSC system was created for patients who experience discomfort and increased fall risk related to their inability to complete simple tasks.

Initial Assessment: The objective pilot study is to provide sufficient evidence that the Voice Smart Care (VSC) System could improve quality of care, patient safety, and patient satisfaction.

Choice of solution: Two [Institution 1] hospitals located in Southern California have piloted the VSC system in their inpatient smart rooms. The VSC study was a randomized design in order to investigate the efficiency and satisfaction of VSC system which uses both voice and touch controls versus the current standard of care provided by nurses.

Implementation: With a simple QR code scan on a smart phone or tablet, the VSC system is activated. The system demonstrates a high level of potential value to the healthcare industry. Patients, families, friends, and nurses can easily control functions from the nursing station and room.

Evaluation: The VSC system utilizes existing hospital technologies and is activated with a simple QR code scan from a smart device. Patients can easily control their environment with verbal or touch commands. [Institution 1] conducted a total of 90 case studies, comprising of $75 \%$ nurse, $20 \%$ patient's family members, and $5 \%$ administrative team.

Interventions: The VSC system assists with questions and requests such as "turn off the light," "adjust the bed," "call nurse," and "turn on the Television". The questionnaire follows Technology Acceptance Model and consists of two different aspects: a 5-star rating and patient comments. Questionnaire results were further analyzed to categorize Star rating 4 and 5 into "AGREE" and star rating 1 and 2 into "DISAGREE".

Lessons learned: In the first question, "I am satisfied with the system and would recommend use it in future," $97.7 \%$ of respondents reported they are satisfied with the system and would recommend its use in the future.

\section{Introduction}

In efforts to improve outcomes and increase quality care, [Institution 1] has begun its journey of utilizing artificial intelligence to enhance healthcare delivery with the Comprehensive Smart Care System. The system will allow patients to use their own smart devices to better access their health care information, control their settings, and grant access to the healthcare professional team after discharge from their inpatient stay. The Comprehensive Smart Care System is a triphasic customized platform which addresses current needs of patients and has the capacity to be built both horizontally and vertically to adopt to the needs and volume of users. The Comprehensive Smart Care System is comprised of three 
segments, and thus is being implemented in three phases. The first phase of implementation to be completed is the utilization of voiceenabled technology. Voice-enabled technology has been widely used in healthcare [1]; however, none have been implemented in the hospital setting [2]. Our study demonstrates the capabilities of a voice control system prototype, in the United States, by using the Voice Smart Care (VSC) System [3]. Voice recognition control, on a tablet or smartphone, is the ability to detect and interpret human speech and trigger a variety of actions [4]. The objective pilot study is to provide sufficient evidence that the VSC system could improve quality of care, patient safety and patient satisfaction.

\section{Case Presentation}

The inspiration for the VSC system came from clinical and personal insights over the past 20-30 years. With the patients' needs in mind and in collaboration with the Internet of Things experts from [institution 2], the VSC system was created. With a simple QR code scan on a smart phone or tablet, the VSC system is activated. Patients, families, friends, and nurses can easily control functions from the nursing station and room. The VSC system assists with questions and requests such as "turn off the light," "adjust the bed," "call nurse," and "turn on the Television". In an evaluation of the new VSC system, a pilot trial was undertaken to assess the impact of VSC implementation. [Institution 1] conducted a total of 90 case studies, comprising of $75 \%$ nurse, $20 \%$ patient's family members, and $5 \%$ administrative team. From this group of 90 respondents, there were $77 \%$ females and $23 \%$ males ranging from $21-80$ years of age. The questionnaire was written from the patient's family members and staff's perception of their experience with the VSC system. Two [Institution 1] hospitals have piloted the VSC system in their inpatient smart rooms. The VSC study was a case study design in order to investigate the efficiency and satisfaction of VSC system which uses both voice and touch controls. The VSC system is available in English, Spanish, Mandarin, and Cantonese.

\section{Discussion}

Our questionnaire follows Technology Acceptance Model (TAM) and consists of two different aspects: a 5-star rating ('1': Strongly Disagree; '2': Disagree;'4': Agree; '5: Strongly Agree) and patient comments. Questionnaire results were further analyzed to categorize Star rating 4 and 5 into "AGREE" and star rating 1 and 2 into "DISAGREE". In the first question, "I am satisfied with the system and would recommend use it in future," $97.7 \%$ of respondents reported they are satisfied with the system and would recommend its use in the future. In addition, $98.1 \%$ of respondents report they agreed this system's interface was easy to operate and use. Moreover, $97.7 \%$ of individuals find the system useful in simple tasks such as controlling lights, Air Conditioner (AC), Television (TV), and bed adjustment. Furthermore, 98.8\% of respondents reported they are satisfied with the VSC system. According to the National Institutes of Health, speech-recognition technology should be adopted and implemented by health care organization as this technology has great impact on the ability of healthcare providers $[5,6]$.

The VSC pilot study has demonstrated improvements in patient satisfaction rates. The system will be gradually implemented hospital wide and to all seven [Institution 1] hospitals to benefit the communities in Southern California. The goal is to make technologies that improve healthcare provider's efficiency and clinical care. In future generations of VSC, the intent is to pair voice recognition apps and machine learning to help diagnose difficult to detect illnesses. A considerable majority of Americans, 95\%, now own a cellphone with at least $77 \%$ having a smartphone [7]. As this number continues to grow in a mobile technology driven era, healthcare should harness this available resource to better serve their patients. The first aspect of the Comprehensive Smart Care System is the admission segment (Figure 1). Smart regulations have an important role in setting checks and balances for emerging technologies [8]. Providing linguistically and culturally appropriate services lend to health promotion [9], as primary and tertiary patient education can bring the patient to maximum health with behavioral changes.

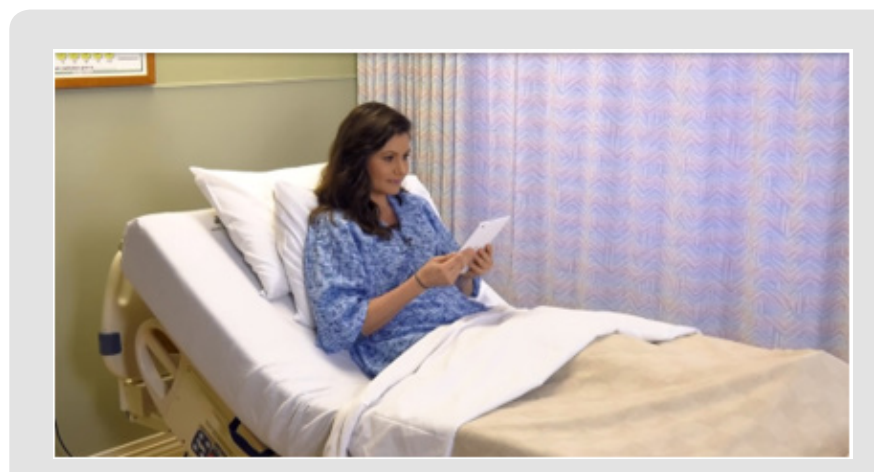

Figure 1: Using the VSC APP to control bed, environment, and communication with nursing station.

The final aspect of the Comprehensive Smart Care System is the discharge segment. Case management can connect patients to their physicians, pharmacists, and other members of the interprofessional healthcare team via text or voice communications. These advancements in access can mimic patient-clinician relationship [10].

Further studies and revision of the system is required in our future study. [Institution 1] will further expand its study with the implementation of the admission and discharge functions of the Comprehensive Smart Care System. The VSC system could advance improvement in quality of care, patient safety, and patient experience.

\section{Acknowledgement}

The authors would like to thank Linda Marsh, David Allen, Kevin Chen, Eing Min Chang, Joseph Badalian, Jon Aquino, Patrick Petre, Iris Lai, Stanley Toy, Charles Dong, Taipei Medical University 
AI Team, Donald Lorack, Paul Hensler, Howard Ternes, Rio Cordova, Nicole Chorvat, Giselle Corral, Jennifer Chang, and Qian Wen.

\section{References}

1. Lu J (2017) The Voice Enabled Revolution. Startup Grind. Medium.

2. Vasyutyn I (2017) Voice AI: Enhance Patient Management \& Healthcare Data. SoftServe.

3. Wen Q (2017) AHMC HealthSystem Voice Smart Care (VSC). YouTube.

4. Kumar A (2017) Opportunities with Voice and AI to Transform Care. Napier Healthcare.

5. Allen J (1982) Implementation of models for speech recognition with very large-scale integrated circuit technology. Automatic Speech Analysis and Recognition: 217-229.

ISSN: 2574-1241

DOI: $10.26717 /$ BJSTR.2020.26.004321

Wen Ta Chiu. Biomed J Sci \& Tech Res

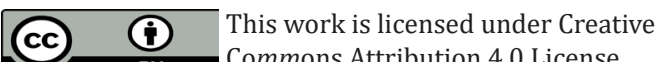

Submission Link: https://biomedres.us/submit-manuscript.php
6. Brunner T (2016) Using technology to give patients a voice after surgery for head and neck cancer. Clinical J Oncology Nursing 20(5): 474-476.

7. (2018) PEW Research Center. Demographics of mobile device ownership - Mobile fact sheet. Pew Research Center.

8. Vogel L (2017) Plan needed to capitalize on robots, AI in health care. Canadian Medical Association 189(8) E239-E330.

9. Lin L (2015) Health care experiences and perceived barriers to health care access: A quality study among African migrants in Guangzhou, Guangdong Province, China. J Immigrant Minority Health 17(5): 15091517.

10. Grant G (2015) Global telehealth 2015: Integrating technology and information for better healthcare. Studies in Health Technology and Informatics 209.

$\begin{array}{ll}\text { BIOMEDICAL } & \text { Assets of Publishing with us } \\ \text { RESEARCHES } & \text { - Global archiving of articles } \\ \text { - Immediate, unrestricted online access }\end{array}$

